\title{
Caroli's Disease: A case report
}

\section{BN Patowary, ${ }^{1}$ S Kumar, ${ }^{2}$ A Pun, ${ }^{3} \mathrm{P}$ Kafle, ${ }^{3} \mathrm{PK}$ Chhetri ${ }^{4}$}

${ }^{1}$ Prof and Head Department of Gastro Surgery . ${ }^{2}$ Mch Resident. ${ }^{3}$ Resident in Surgery, ${ }^{4}$ Assistant Prof Department Radiodiagnosis, College of Medical Sciences, Bharatpur, Nepal

\begin{abstract}
Caroli's disease $(C D)$ is a rare congenital abnormality characterized by dilatation of intra-hepatic bile ducts, recurrent cholangitis, formation of calculi inside these ducts with normal extra hepatic ducts and higher risk for cholangiocarcinoma. Association of this disease with congenital hepatic fibrosis is named as Caroli's syndrome. We reported 30 years old women with recurrent epigastric pain for 2 years with normal Liver function Test however involving both lobes of liver who developed cholangiocarcinoma approximately 6 months later.
\end{abstract}

\section{Key Word: Carolis disease, congenital hepatic fibrosis , cholangiocarcinoma.}

\section{Introduction}

Caroli's disease was first described by Caroli as a congenital malformation of intrahepatic bile ducts,characterized by segmental cystic dilatation of the intra hepatic duct;increased incidence of biliarylithiasis; cholangitis, Liver abscess, absence of cirrhosis and portal hypertension; and association with renal tubular ectasia or similar renal cystic disease. ${ }^{1}$ Association of this anomaly with congenital hepatic fibrosis and portal hypertension is named as Caroli's syndrome. This disease affects about 1 in 10, 00000 people with more reported cases of Caroli's syndrome than Caroli's disease. Caroli's disease is typically found in Asia and under 22 years of age. The cause of the disease appears to be genetic, while Caroli's disease is an autosomal dominant trait; the complex Caroli's

Correspondence: Dr. BN Patowary

E-mail: bhupendra_patowary@yahoo.com syndrome is an autosomal recessive trait. Caroli's disease is distinct from other diseases that causes ductal dilatation caused by obstruction in that it is not one of the many choledocal cyst derivatives. ${ }^{2}$ Incidence of Females to male ratio is $4: 1{ }^{3}$ There is a link between Caroli's disease and autosomal recessive polycystic kidney disease. ${ }^{4}$ First symptoms typically include fever, intermittent abdominal pain and hepatomegaly. Occasionally jaundice appears due to cholangitis. Computed Tomography scan can confirm the diagnosis. However, X-ray, MRI will show enlarged intra-hepatic bile ducts due to ectasia. USG is also helpful showing the tubular dilatation of the bile ducts. A high contrast CT scan can distinguish between intra-hepatic stones and dilated ducts. However, cholangiography is the best and final approach to show the enlarged bile ducts of Caroli's disease. ${ }^{5}$ 
Figure 1: Diagrammatic representation of intrahepatic bile ducts dilatation

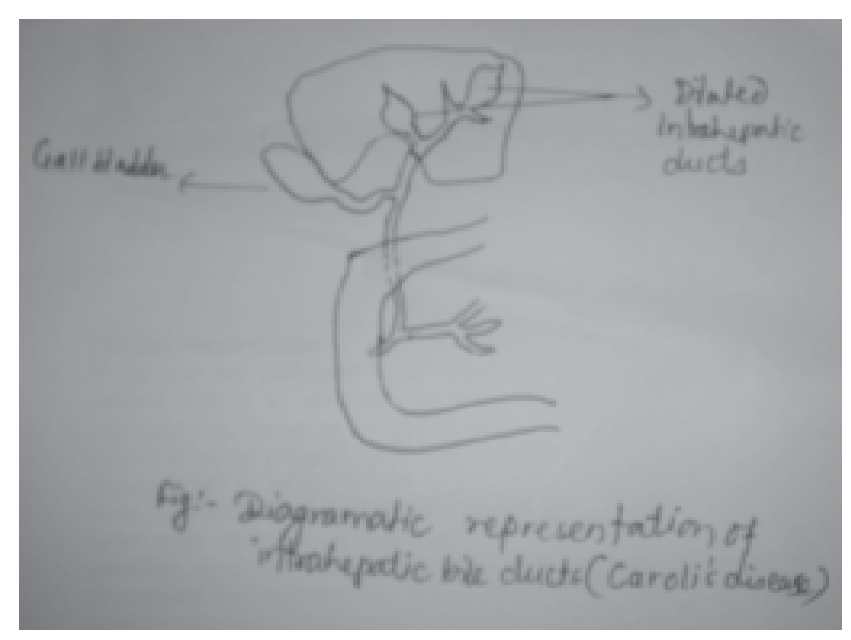

Figure 2: Computed tomography demonstrating intrahepatic biliary dilatation (Arrow head)

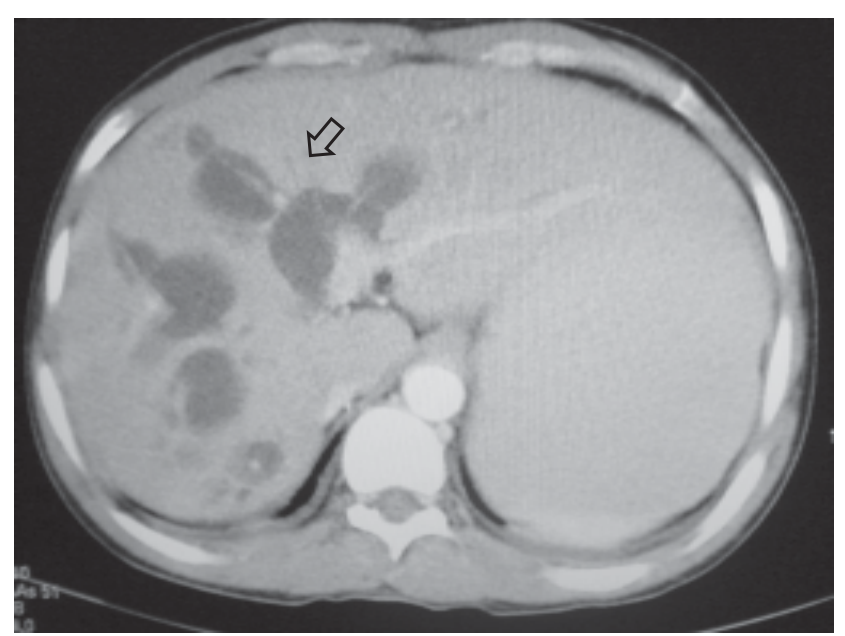

Treatment depends on the clinical feature and location of the disease. When one lobe is affected lobectomy relieves the symptoms and removing the future risk of malignancy. $7 \%$ of Caroli's disease can develop cholangiocarcinoma. ${ }^{2}$ Antibiotics may be used in presence of cholangitis and ursodoxycholic acid for hepatolithiasis. Approximately 68\% of the affected persons develop biliary lithiasis. ${ }^{6}$ In cases of migration of stone into extra hepatic bile ducts Endospic
Retrograde Pancreatico-cholangiography removal of stone or sphincterotomy can be done. Liver transplantation can be done in bilaterally involved cases. $^{7}$

\section{Case report}

A 30 years female from remote place of Tanahu, presented with intermittent epigastric pain and fever for 2 years. Persistent abdominal pain in the epigastric region was hospitalized and treated by hydration, antibiotics and analgesics. She also gave the history of jaundice (off/on) since 2 years. At the beginning, attacks have occurred every 6-7 months but progressively the intervals have shortened to 4 weeks for last 3 months. She noticed loss of weight (5-6 kg), nausea, vomiting and constipation and anemia. Physical examination revealed tenderness in upper abdominal quadrants with no lymphadenopathy and organomegaly. Because of recurrent epigastric pain, physical findings and anemia we decided to performe sophagogastroduodenoscopy (EGD) and abdominal ulttrasonography (USG). Endoscopic evaluation showed antral gastritis with duodenitis. Pathological study of second part of duodenum demonstrated features suggestive of chronic duodenitis. Abdomenal ultrasound showed cystic dilatation of intrahepatic biliary ducts of both lobes of liver with calculi. So Contrast enhanced computed tomography of abdomen was planned which showed findings similar to that in ultrasoundgraph while extra hepatic ducts were normal. There was tiny splenic infarction present. No organomegaly was seen. Further diagnostic procedures were not performed because the computed tomography was considered sufficient. 
Figure 3: Computed tomography of abdomen demonstrating the liver secondaries (Arrow heads)

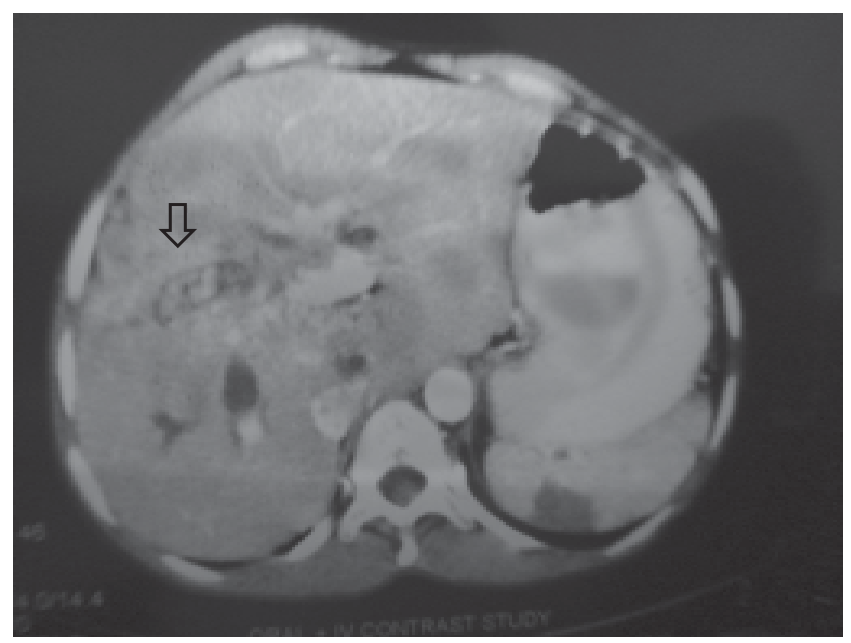

Laboratory findings: On evaluating the LFT, TLC, DLC Platelet Renal function tests, PT and INR, Serum Carcinoembryonic Antigen all were within the normal limits. With the above finding without renal dysfunction, coagulation disorder, portal hypertension and malignancy she was managed conservatively with broad spectrum antibiotics, intravenous fluid and analgesic. She responded well to medical management. Discharged from the hospital and was adviced for follow up every 3 months. During her initial check- up she didn't have further complaint except occasional right epigastric and right upper quadrant abdominal pain of low intensity. Later, she became irregular in her check up. On her second follw up she complained of anorexia, nausea, vomiting, abdominal pain and deep jaundice. On examination she had evidence of malignancy in the liver. CT scan of abdomen examination revealed Carcnomatous change in the right lobe of the liver

\section{Discussion}

Diagnosis of Caroli's disease is based on clinical features and imaging studies. The normal course of the disease consists of suppurative cholangitis, septicemia with gram-negative organisms and intra-hepatic abscesses. Because of its rarity diagnosis of the disease is often delayed. Contrast CT scan confirm the diagnosis by showing intrahepatic dilatation of billiary tree with calculi inside. Other diagnostic imagings are not required. In our case, diagnosis could easily be made by CECT and so we didn't feel the necessity of MRI or ERCP. It has been shown that magnetic resonance cholangio-pancreatography (MRCP) is an appropriate diagnostic tool and equivalent to ERCP in detecting and defining the morphologic characteristics of congenital cyst lesions of the bileduct. The sensitivity and specificity of MRCP are $82 \%$ and $100 \%$ respectively. ${ }^{8}$ Modified Todani Classification has included Caroli's disease under Type V Choledochal cyst. The classical triad of pain in right upper abdominal quadrant, mass and jaundice is present in only $10 \%$ of cases of Choledochal cyst. ${ }^{9}$ Besides,In Caroli's disease jaundice is usually not seen. Clinical presentation of jaundice in Caroli's disease is due to presence of cholangitis.

It is necessary to demonstrate that the Caroli's disease is localized or diffuse from the point of view of treating the disease. Caroli's disease if localized to one lobe, can be treated with lobectomy, not only to get rid of the disease but also to prevent malignant transformation. Removal of calculi and sphincterotomy may be needed when calculi have migrated into the extra-hepatic bile ducts.Hepatic transplantation is indicated for the treatment of diffuse type of caroli's disease. ${ }^{10}$ Because of the characteristic appearance of the bile ducts and frequent association with renal tubular ectasia, the differential diagnosis of Caroli's disease is limited. At times, cholangitis may result in intrahepatic abscesses and communicate with the bile ducts and mimic Caroli's disease. However, the margins of the abscess wall are irregular unlike ductal walls of Caroli's disease. 
Journal of College of Medical Sciences-Nepal, 2012, Vol-8, No-3

Primary sclerosing cholangits may come to the differential diagnosis of Caroli's disease since PSC also demonstrate biliary dilatation and may be associated with intra-ductal calculi. ${ }^{11}$

Cholangiocarcinoma which occurs in $7 \%$ of cases is a fatal complication. ${ }^{2}$ In our case while we did a regular follow up of our patients, we didn't come across any other complications. But after about 6 months when she came for a follow up, she had considerable loss of weight, jaundice, anorexia and distension of abdomen. We therefore, decided to exclude malignant change and subjected her for another CECT and we found that she already had cholangiocarcinoma. Although the case was still operable we couldn't either treat her in our hospital due to lack up infrastructure facility, nor could we refer her elsewhere as the patient was very poor to afford such a treatment.

\section{Conclusions}

Though Caroli's disease is congenital in origin, manifest late as adulthood or middle age. The classical clinical features are not always present. Caroli's disease should be considered in differential diagnosis of patients with recurrent epigastric and / or right upper quadrant abdominal pain even without jaundice in this age group. CECT abdomen is a useful tool for its diagnosis. Endoscope retrograde cholangiography and Magnetic Resonace Cholangiography are required in cases of diagnostic dilemma. Even though it is a rare disease high index of clinical suspicious is required. Proper clinical evaluation and radiological investigation is adequate for the diagnosis of Caroli's disease. We must exclude its association with Renal disease. Portal hypertension and coagulation disorder. Cholangiocarcinomatous change should be kept in mine.

\section{Reference}

1. Bayraktar Y.Clinical characters of Caroli's disease.World J Gastroenterol 2007; 13(13):1930-33.

2. Karim AS. Caroli's Disease Case Reports. Indian Pediatr.2004; 41(8):848-50.

3. Nagorney DM. Bile duct cysts in adults. In: Blumgart LH, Fong Y (eds) Surgery of the liver and biliary tract, 3rd edn.Saunders, London, 1229-44.

4. Friedman JR: Caroli Disease at eMedicine. http:// www.micopedia.org/mickify.py?topic $=$ caroli_disease. Assessed July 16, 2012.

5. Chiba T. , Shinozaki M, Kato M, et al. Caroli's disease: central dot sign re-examined by CT arteriography and CT during arterial portography , Eur Radiol: 2002.12 (3): $701-2$

6. Guma C, Viola C, Apestegui M, et al. Hepatolithiasis and Caroli's disease in Argentina: results of a multicenter study. Acta Gastroenterol Latinoam. 1999; 29(1):9-15.

7. Ulrich F, Steinmuller T, Settmacher U, et al.Therapy of Caroli's disease by orthotopic liver transplantation. Transplant. Proc. 2002; 34 (6): 2279-80.

8. Zhong L, Xiao SD, Stoker J, et al. Magnetic resonance cholangiopancreatography. Chin J Dig Dis. 2004; 5 (4) : $139-48$

9. Malangoni MA, Rosen MJ. Hernias ,Sabiston Text Book of Surgery the Biological Basis of Modern Surgical Practice, $18^{\text {th }}$ edi. Philadelphia ,Elsevier Saunders. 2008:1155-79.

10. Bratu BL, Labergte JM, Khalife S, et al. Regression of antenatally diagnosed localized Caroli's disease. $J$ Pediatr Surg. 2000; 35 (9): 1390-3

11. Fulcher AS, Mary AT, Arun T, et al. Caroli disease and Renal Tubular ectasis. Radiology .2001 ;220(3): 720-23. 